\title{
Electromigration-induced Plasticity and Texture in Cu Interconnects
}

\author{
A. S. Budiman^ ${ }^{\wedge}$ C.S. Hau-Riege ${ }^{\wedge}$ P.R. Besser ${ }^{\wedge}$, A. Marathe ${ }^{\wedge}$ \\ Y.-C. Joo^ N. Tamura J.R. Patel`^^ W.D. Nix^${ }^{\wedge}$ \\ 'Dept. Of Materials Science \& Engineering., Stanford University, Stanford, CA 94305 \\ $\wedge$ AdvancedMicro Devices, Inc., Sunnyvale, CA \\ $\wedge$ Dept Of Materials Science \& Engineering, Seoul National University (SNU) \\ "AdvancedLight Source (ALS), Lawrence Berkeley National Laboratory (LBNL),Berkeley, CA 94720
}

\begin{abstract}
Plastic deformation has been observed in damascene $\mathrm{Cu}$ interconnect test structures during an in-situ electromigration experiment and before the onset of visible microstructural damage (ie. voiding) using a synchrotron technique of white beam X-ray microdiffraction. We show here that the extent of this electromigration-induced plasticity is dependent on the texture of the $\mathrm{Cu}$ grains in the line. In lines with strong < 111> textures, the extent of plastic deformation is found to be relatively large compared to our plasticity results in the previous study [1] using another set of $\mathrm{Cu}$ lines with weaker textures. This is consistent with our earlier observation that the occurrence of plastic deformation in a given grain can be strongly correlated with the availability of a $<112>$ direction of the crystal in the proximity of the direction of the electron flow in the line (within an angle of $10^{\circ}$ ). In $<111>$ out-of-plane oriented grains in a damascene interconnect scheme, the crystal plane facing the sidewall tends to be a $\{110\}$ plane,[2-4] so as to minimize interfacial energy. Therefore, it is deterministic rather than probabilistic that the $<111>$ grains will have a $<112>$ direction nearly parallel to the direction of electron flow. Thus, strong $<111>$ textures lead to more plasticity, as we observe.
\end{abstract}

\section{INTRODUCTION}

Most interconnect metals are aggregates of crystalline grains. The crystalline lattice of each grain has a characteristic orientation, and a polycrystal is thus characterized by a distribution of orientations - its texture. Texture governs many of the physical, electrical and mechanical properties of polycrystalline materials. In metallic conductor hues in microelectronics integrated circuits, texture has been known to play important roles in the performance and reliability of the conductors, for instance in electromigration. [5]

Recently, an unexpected mode of plastic deformation has been observed in Al[6,7] as well as in $\mathrm{Cu}[1,8,9]$ conductors - or intercormects, as they are usually referred to in industry - during electromigration (EM) stressing. This deformation occurs not in any random direction, but always in a direction transverse to the direction of electron flow in the lines. Texture and crystal plasticity have a close, intertwined relationship;Textures evolve during plastic deformation, while plastic deformation often depends

on crystallographic orientations. Understanding crystal plasticity and how it progresses in intercormect lines during electromigration therefore might lead us to fundamental insights on the role of texture in electromigration. 
The grains that exhibit plastic deformation were always observed to deform (bend, rotate or polygonize) transverse to the direction of the electron flow in the line, or in other words, across the width of the hne. $\wedge^{\wedge^{\prime}} \wedge^{\prime \prime}$ We further observed, especially in $\mathrm{Cu}$ hues, [1,9] that the occurrence of plasticity can be correlated with the availability of a $<112>$ direction in the proximity of the direction of the length of the line (within $10^{\circ}$ ).

In the present study, a different set of $\mathrm{Cu}$ hues fabricated by a different manufacturer is investigated. This set of $\mathrm{Cu}$ lines differs with the previous set[l,8,9] in a few ways; chief among them is texture. Using the synchrotron technique of white beam X-ray microdiffraction,[10] developed in Beamhne 7.3.3 at the Advanced Light Source, Berkeley Lab, we follow the evolution of plasticity in Cu polycrystals during similar electromigration experiments. A strong texture dependence is observed and a model is proposed to explain the prominent observation.

\section{EXPERIMENTAL}

The intercormect test structure used in this study is a variation of a BEoL process for a 65rmi CMOS technology, as shown Figure 1.

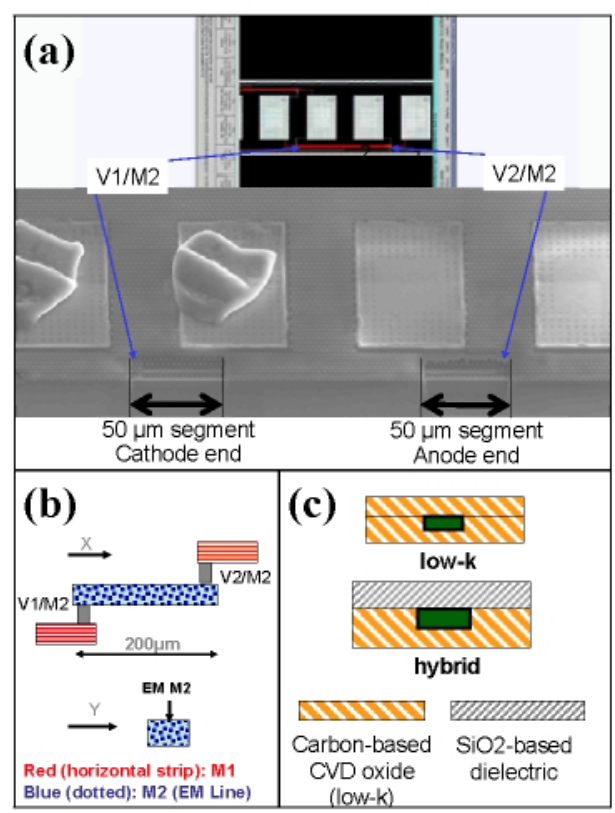

FIGURE 1. Cu interconnect test structures manufactured by AMD; (a) SEM image of the test structure (Acknowledgement: Bryan Tracy of Spansion); (b) in situ electromigration experiment; (c) two sets of test structures of different dielectric schemes: low-A: vs hybrid. 
The M2 lines were studied after partial removal of the top dielectric to expose the capped $\mathrm{Cu}$ hnes (Figure 1(a)). In this technology, the $\mathrm{Cu}$ fill process includes a standard Ta-based barrier and $\mathrm{Cu}$ seed, electroplated $\mathrm{Cu}$ fill, post-plating anneal, chemical-mechanical polish and a dielectric cap layer. Two different inter layer dielectrics (ILD) were integrated with copper: $\mathrm{Cu} / \mathrm{low}_{-} \wedge$ ILD (Low- $\wedge$ = CVD carbondoped oxide) and $\mathrm{Cu} /$ Hybrid ILD (Hybrid $=\mathrm{Cu} / \mathrm{low}^{-\wedge}$ at the line level and $\mathrm{Cu} / \mathrm{FTEOS}$ at the via layer). Both ILD materials were studied in order to provide a comparison of the extent of plasticity.

The first set of test structures consists of lines 200 im length, approximately 0.2 |im thick, and 0.5 |im wide. Due to limited beam time, typical of synchrotron experiments, only segments of 50 |im length at both cathode and anode ends of the hne were studied (Figure 1(a)). The dielectric is carbon-based CVD oxide ("low- $\wedge$ " in Figure 1(c)). The second set of intercormect test structures was prepared with dimensions similar to those of the first one, but with the hybrid ILD material (Si02 based). The line length is 200 |im, the thickness is approximately 0.25 |im and the width is $0.7 \mathrm{im}$. Similarly, only segments of $50 \mathrm{~nm}$ length at both cathode and anode ends of the hne were studied.

The synchrotron technique of scarming white beam X-ray microdiffraction has been described completely in a previous pubhcation.[IO] The experiment was performed on beamline 7.3.3. at the Advanced Light Source, Berkeley, CA. The electromigration test was conducted at $300^{\circ} \mathrm{C}$ on a via-terminated test structure (Figure 1(b)). Current and voltage were monitored at lOs increments. The sample was scarmed in $0.5 \mathrm{im}$ steps. A complete set of CCD frames takes about 6 to 7 hours to collect. The exposure time was $20 \mathrm{~s}$ plus about $10 \mathrm{~s}$ of electronic readout time for each frame. In this maimer the Laue pattern and information regarding plastic deformation for each grain in the sample was collected for each time step during the experiment. The current was ramped up to $2 \mathrm{~mA}\left(/^{\prime}=2 \mathrm{MA} / \mathrm{cm}^{\wedge}\right)$ and then set at that value for the rest of the test (up to 36 hrs).

\section{RESULTS AND DISCUSSION}

We first describe the in situ electromigration observations on both of the $\mathrm{Cu}$ damascene test structures.

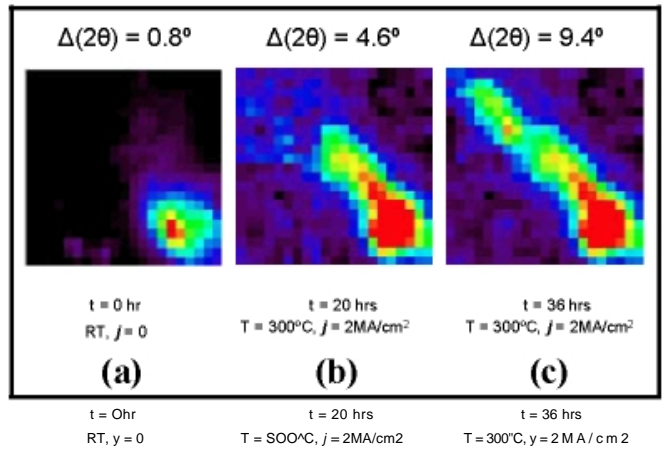

FIGURE 2. The typical evolution of Laue diffractions spots from $\mathrm{Cu}$ interconnect test structures during in situ electromigration experiments. 


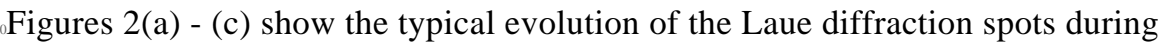
the in-situ EM test. Figure 2 is early in the EM test, sampled after 36 hours of testing. The observed broadening of the Laue diffraction spots (streaking) represents plastic deformation of the $\mathrm{Cu}$ grains induced by EM.

\section{Electromigration-induced Plasticity: The Directionality}

As the EM test progresses, plasticity is observed in the $\mathrm{Cu}$ grains throughout the hue, such as demonstrated in Figure 3(a). Plasticity here may manifest itself either in the form of diffraction spot broadening (streaking) or in the form of diffraction spot splitting into two or even more different spots. The broadening of the diffraction spots represents crystal bending of the $\mathrm{Cu}$ grains in the line, whereas the split diffraction spots indicate the formation of low-angle boundaries or sub-grain structures. From the amount of broadening the bending of the $\mathrm{Cu}$ crystal can be calculated. From the amount of splitting, the angle of misorientation can be determined.

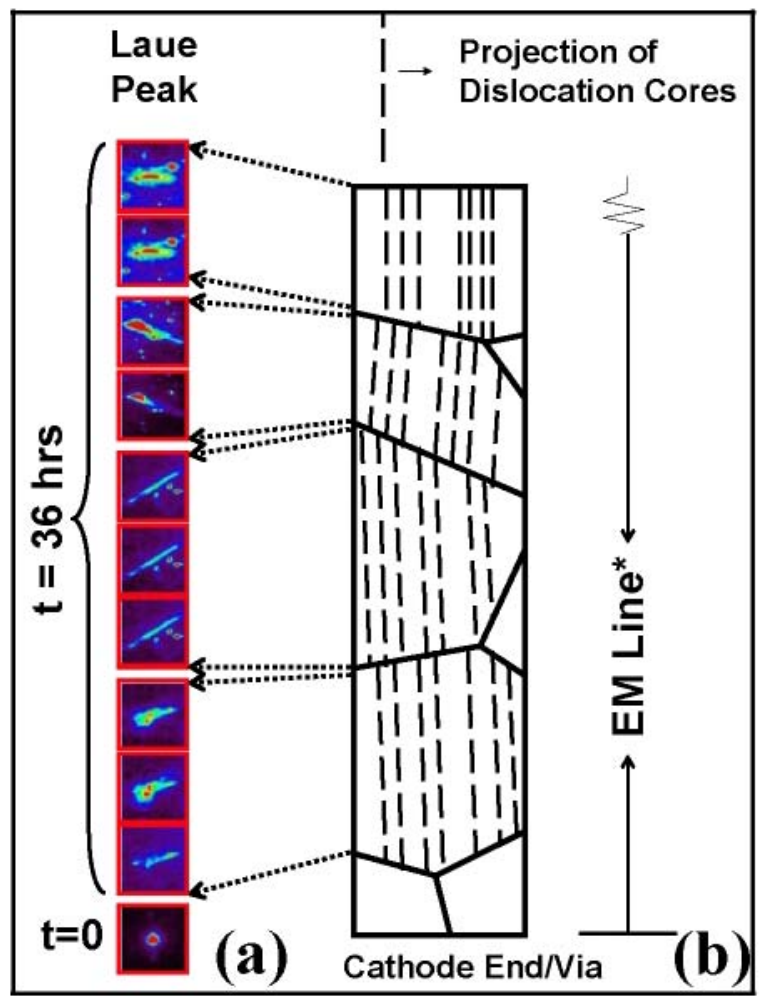

FIGURE 3.(a) Streaking and/or splitting of Cu Laue diffractions spots throughout a segment of the hne; (b) Dislocations were found with cores lining up with the direction of the electron flow in the line (consistent with our earlier observation"*') across grains throughout the length of the segment of the line observed (*Grain map is estimated based on streaking observation). 
${ }$ Not only was plasticity observed, but also the direction of the plastic deformation is generally consistent across grains throughout the segments of the line under observation, as shown in Figure 3(a). This is consistent with our observation on the previous set of $\mathrm{Cu}$ lines. $[1,8,9] \mathrm{Cu}$ grains plastically deform in a direction transverse to the electron flow direction in the hue. Such directionality can simply be accommodated by a distribution of same sign edge dislocations with cores as illustrated in Figure 3(b), ie. with the $<112>$ hue direction of the dislocations are all lining up nearly to the direction of electron flow in the line.

Grain orientation mapping of these $\mathrm{Cu}$ hues unfortunately could not be obtained in the present study. The X-ray spot size $(0.8$ x $0.8 \mathrm{lim})$ currently used in ALS beamline 7.3.3 was relatively large for the dimensions of these state-of-the-art intercormect lines. That makes diffraction spot indexation often very difficult and thus mapping of grain orientations and other further quantitative analyses unreliable. The few $\mathrm{Cu}$ grains that we show in Figure 3 were among the limited number of grains in the two $\mathrm{Cu}$ lines for which indexation of the diffraction spots happen to be sufficiently clear and unambiguous for this analysis. In general, the larger the $\mathrm{Cu}$ grains, and the more bamboo-like they are, the more they diffract sharply and give numerous diffraction spots, thus giving higher confidence on the reliability of these results. That being said, it is fortunate that we can still always compare qualitatively the evolution of $\mathrm{Cu}$ diffraction spots before and after some period of EM testing, as demonstrated in Figure 2.

\section{Electromigration-induced Plasticity: The Extent}

The extent of the EM-induced plasticity observed in the present samples will now be determined. Figures 4(a) and (b) show still different additional diffraction spots observed during this experiment (after EM testing of 36 hours, at $300^{\circ} \mathrm{C}$ and $2 \mathrm{MA} / \mathrm{cm}^{\wedge}$ current loading) coming from the $\mathrm{Cu}$ hues with the low- $\wedge$ and the hybrid dielectrics, respectively. The diffraction spots have been converted to $x-\wedge()$ space, with / rurming along the direction of the length of the line, and 20 across the direction of the width of the line.

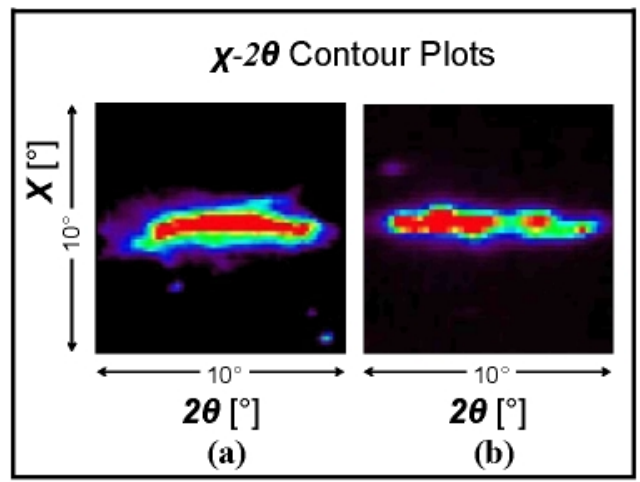

FIGURE 4. The dielectric effects: the Laue peak streaking/sphtting observed from Cu interconnect test structures with (a) low-A:, and (b) hybrid dielectrics; in x-2(? space/contour intensity plot. 
oWe can then use the broadening and the spot sphtting observed to obtain information about the dislocation structure induced into the grain by electromigration. For instance, from the streak length of Figure 4(a), as measured in the CCD camera and the sample to detector distance, we determine the curvature angle of the grain of $9.8^{\circ}$. Assuming a near bamboo structure, the grain width is the same as the width of the hue $\left(0.5^{\wedge} \mathrm{m}\right)$, from which we detemine the radius of curvature of the grain, $R=$ $2.34 \mathrm{~nm}$. The geometrically necessary dislocation density needed to account for the curvature observed can be calculated from the Cahn-Nye relationship, [11,12] $p=l / R b$ where $b$ is the Burgers vector. The geometrically necessary dislocation (GND) density is then $p=1.68 \mathrm{xlo}^{\prime} \wedge \mathrm{W}$. The total number of dislocations in the area of the crosssection of the $\mathrm{Cu}$ line/grain is approximately 142 .

To obtain quantitative information on polygonization walls (subgrain boundaries) from the spot split in Figure 4(b) we observe that the Laue spot splitting, Atotai $=9.1^{\circ}$. From this misorientation and Burgers' model of a small angle grain boundary $6=b I L$, where $L=$ dislocation spacing, we find $\mathrm{Z}=16$ A which amounts to a total of 110 dislocations in the subgrain boundaries in the cross-section of the $\mathrm{Cu}$ line/grain. This translates to the GND density of $\mathrm{p}=1.12 \mathrm{xio} / \mathrm{N} / \mathrm{ml}$

The extent of the plasticity as described here $\left(\mathrm{o} \sim 1 \mathrm{O}^{\prime} \wedge \mathrm{W}\right)$ is observed across all grains throughout the segments of both hues with different dielectric schemes. The significance of the difference in our analysis above, in terms of the extent of the plasticity, as well as, its manifestation (grain bending vs polygonization) between the two $\mathrm{Cu}$ lines with different dielectric schemes requires added confirmation. Nevertheless they provide a general indication of the extent of plasticity in these $\mathrm{Cu}$ hues.

\section{Proposed Correlation: Texture vs. EM-induced Plasticity}

Compared to the typical observation of the extent of the EM-induced plasticity in the previous set of $\mathrm{Cu}$ intercormect lines,[1,8,9] this set of samples exhibits at least a two-order of magnitude difference, in terms of GND density (Figure 5). The former sample exhibited $p \sim 1 \mathrm{O}^{\prime} \wedge-10^{\prime} \wedge \mathrm{W}$ (from here onwards, we call these "Samples A"), and the latter exhibited $p \sim 1 \mathrm{O}^{\prime} \wedge \mathrm{W}$ ("Samples B"). As a reminder. Samples A and B were fabricated by different manufacturers and differ fairly significantly in dimensions, as well as the dielectric materials used, as shown in Figure 5 (the dimensions are to scale).

Figure 5 shows the typical evolution of the Laue reflections from the $\mathrm{Cu}$ lines from the initial state $\left(\mathrm{RT}, \_/=Q, t=\right.$ time $=0$ ) to the EM state (after some electromigration, $T$ $=300^{\circ} \mathrm{C}, 7-2.0-3.1 \mathrm{MA} / \mathrm{cm}^{\wedge}, \wedge \sim 36-96 \mathrm{hrs}$ ). Care was taken in order for the observed intensity contours in the $x$ - 20 coordinate in Figure 6 to be comparable (the windows all cover areas of squares of a range of $7^{\circ}-10^{\circ}$ in angle space, and the threshold of the lower-bound intensity display was set to be similar). Thus it is obvious from the relative apparent difference in the extent of streaking/splitting of the Laue diffraction spots that the level of plastic deformation that developed during the course of electromigration in Samples B is distinctly larger than that of Samples A. 


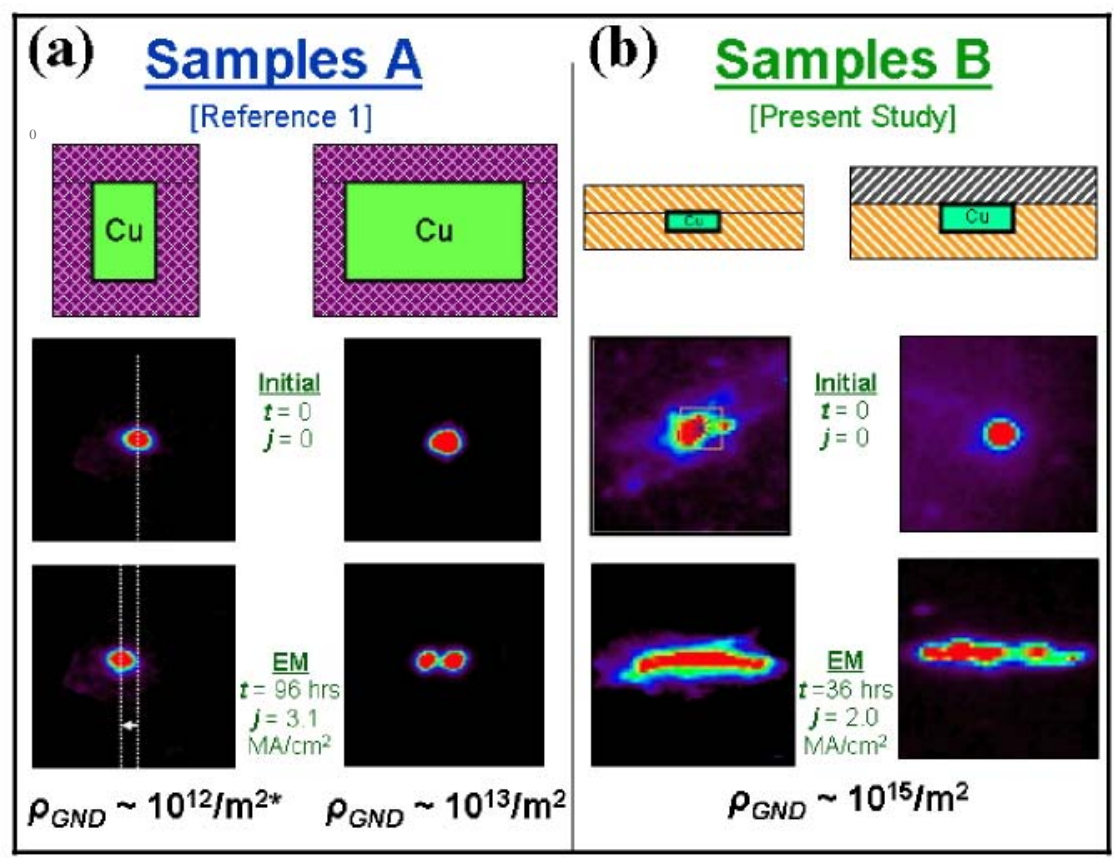

FIGURE 5. Samples A vs B comparison: the schematic of the cross-sections (color code refers to different materials), typical evolution of Cu Laue diffraction spots (from "Initial" to "EM" states), and lastly, the typical densities of GNDs implied by the extent of streaking/splitting of Laue peaks; (a) Samples A (Cu lines reported in our previous studies"*'); (b) Samples B (Cu lines reported in this study); They were fabricated by different manufacturers. (*taken as that of typical annealed metals)

As the two sets of samples (Samples A vs B) under investigation are provided by different integrated circuit manufacturers, it is not possible to completely quantify the process differences (dielectric type, materials processing and thermal history) in their technologies in this publication. It is known that the two sets of samples differ in terms of dimension and dielectric materials used; however, it is assumed that the main difference, as far as EM-induced plasticity is concerned, is the crystallographic texture of the $\mathrm{Cu}$ lines. From the texture analysis conducted in our previous study, [8] we learn that Samples A have a rather weak (111) texture. Meanwhile, Samples B came from the same manufacturer of the inlaid $\mathrm{Cu}$ lines studied by Besser et al. [2] It is therefore reasonable to assert that Samples B would have the typical strong (III) texture observed by Besser et al.[2] Unfortunately, a grain orientation mapping using Beamhne 7.3.3 in the present study was not possible for Samples B for reasons that have been discussed in the earlier part of this manuscript.

While other process and dimension differences between these two sets of samples include dimensions and dielectric materials are acknowledged, we believe that these differences carmot satisfactorily explain the differences in the extent of plastic deformation. For example, the $\mathrm{Cu}$ in Samples $\mathrm{A}$ is surrounded completely by dielectric material, which is a fluorinated Si02-based dielectric, and thus generally believed to constrain the $\mathrm{Cu}$ lines better and should result in less plastic deformation. This is 
consistent with our observation of Samples A vs Samples B, but the different dielectric schemes in Samples B, do not appear to affect the level of plasticity in the $\mathrm{Cu}$ lines. Another example involves the size effect. Wider lines seem to exhibit more plastic deformation in our previous study,[1,8,9] such as shown also in Figure 6(a). However, Samples B actually are much narrower, and also much smaller in all crosssectional dimensions than Samples A, but yet Samples B exhibits two orders of magnitude more EM-induced plasticity.

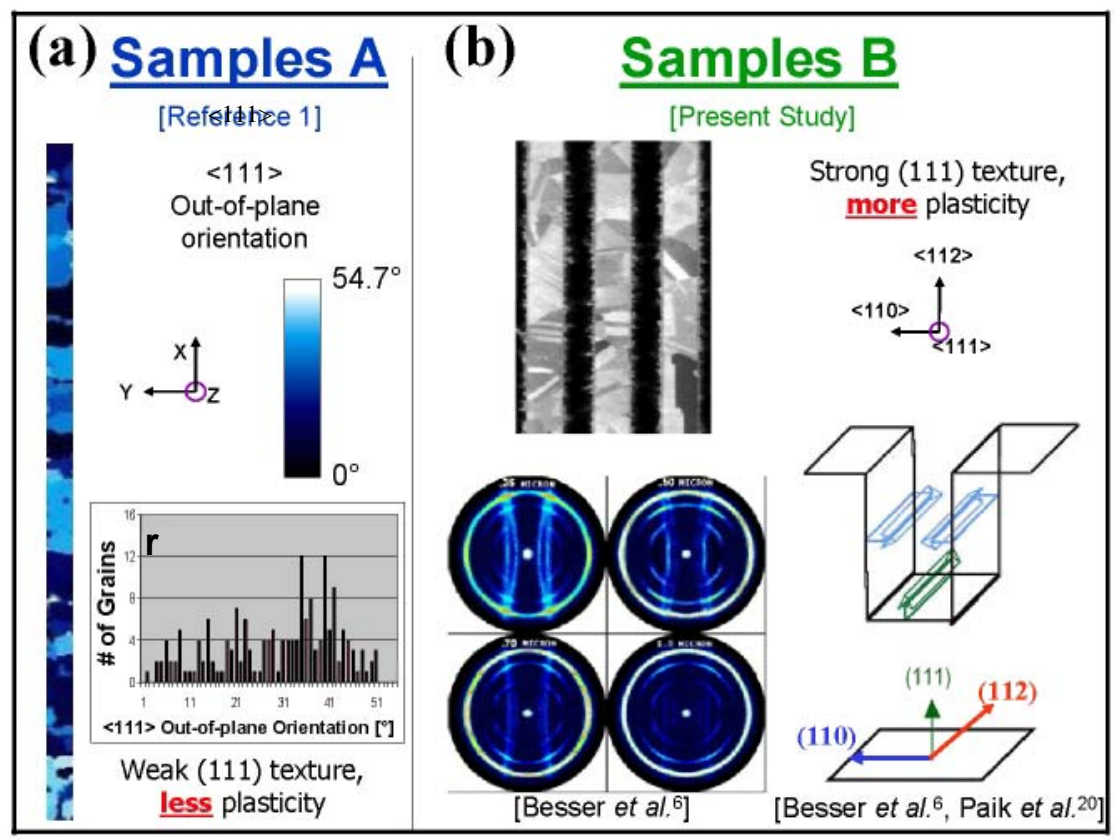

FIGURE 6. Samples A vs B comparison: the texture correlation; (a) Samples A (Cu lines reported in our previous studies"*') have weak (111) texture, and "less plasticity," whereas; (b) Samples B (Cu lines reported in this study) have strong (111) texture, and "more plasticity"; Illustration of strong preferred in-plane orientation of (111) grains: $<110>$ to the sidewalls, $<112>$ along the direction of length of the lines (Courtesy of Besser $e t a^{\wedge}$ )

Figure 6 is a comparison summary of the known information about the $\mathrm{Cu}$ lines in Samples A vs Samples B. First, Samples A shows a weak (111) texture, and we found the extent of EM-induced plasticity in the order of $p \sim 1 \mathrm{O}^{\prime} \wedge-1 \mathrm{O}^{\prime} \wedge \mathrm{W}$. Subsequently, Samples B was found with $p \sim 1 \mathrm{O}^{\prime} \wedge \mathrm{W}$ after similar electromigration conditions - a significantly larger amount of EM-induced plasticity. Besser et al. [2] suggested that Samples B have the typical strong (111) texture. This observation of significantly larger EM-induced plasticity in Samples B compared to that of Samples A, thus, is consistent with our earlier observation, especially detailed in Reference 9, that the occurrence of plastic deformation in a given grain can be strongly correlated with the availability of a $<112>$ direction of the crystal in the proximity of the direction of the electron flow in the line (within an angle of $10^{\circ}$ ). In $<111>$ out-of-plane oriented 
grains in a damascene interconnect scheme, the crystal plane facing the sidewall tends to be a $\{110\}$ plane, so as to minimize the interfacial energy.[2-4] Therefore, it is deterministic rather than probabilistic that the $<111>$ grains will have a $<112>$ direction nearly parallel to the direction of electron flow or the direction of the length of the line. This is illustrated in Figure 6(b).

In Samples B, most grains are $<111>$ in out-of-plane orientation (such as shown in the FIB mapping in Figure 6(b)), and thus prefer energetically to have the $<110>$ directions normal to the sidewalls, thus causing a $<112>$ direction to be very close to the direction of the electron flow. When this condition is met, our proposed correlation, discussed earlier in our previous studies, $[1,8]$ but more in detailed maimer in Reference 9, suggests that plasticity occurs in these $\mathrm{Cu}$ grains upon electromigration, and not only did it occur, the extent of the plasticity here was rather extreme. Samples $\mathrm{A}$, in the meantime, have only a few grains that are $<111>$ in out-of-plane orientation, which led to the occurrence of plasticity only in these few grains in the $\mathrm{Cu}$ lines after electromigration. In most other grains (ie. non $<$ lll $>$-oriented grains), a $<112>$ direction of the $\mathrm{Cu}$ crystal is likely to be not in the direction of the electron flow of the hues. This is the reason plasticity was not observed in many grains in the $\mathrm{Cu}$ lines of Samples A.

\section{CONCLUSIONS}

In conclusion, we have further observed plastic deformation behavior of $\mathrm{Cu}$ polycrystals during electromigration experiments, using a synchrotron technique involving white-beam X-ray microdiffraction. With the present set of $\mathrm{Cu}$ lines, we further confirm the nature of the plastic behavior observed in these $\mathrm{Cu}$ hues: the direction of the plastic deformation and the availability of a $<112>$ direction along the length of the lines as the rotation axis of the plastic deformation. Furthermore, we found that the extent of the electromigration-induced plasticity in this set of $\mathrm{Cu}$ lines was significantly larger compared to that of the previous study. We propose that the crystallographic texture of the $\mathrm{Cu}$ lines plays a primary role in controlling the plastic behavior of the intercormect lines. Strong (111) texture leads to high preference of $<112>$ direction along the length of the line, and this subsequently leads to higher tendency for the grains to behave plastically in respond to electromigration stressing.

\section{ACKNOWLEDGMENTS}

The authors would like to thank Advanced Micro Devices (AMD) for generous support and sample assistance. One of the authors (ASB) more specifically would like to thank John M. Ennals, AMD/SRC Program Manager, Technology Research Group, AMD, for the opportunity of a Summer Internship Program in 2006. Both ASB and WDN gratefully acknowledge support by the U.S. Department of Energy, Office of Basic Energy Sciences through Grant No. (DE-FG02-04ER46163). The Advanced Light Source (ALS) is supported by the Director, Office of Science, Office of Basic 
¿Energy Sciences, of the U.S. Department of Energy under Contract No. DE-AC0205CH11231 at the Ernest Orlando Lawrence Berkeley National Laboratory (LBNL).

\section{REFERENCES}

1. A. S. Budiman, N. Tamura, B. C. Valek, K. Gadre, J. Maiz, R. Spolenak, W. D. Nix and J. R. Patel, Appl. Phys. Lett. 88, 233515 (2006).

2. P. Besser et al., J. Elec. Math., 30, 4, 320 (2001).

3. J.E. Sanchez, Jr. and P.R. Besser, Proceedings of the International Interconnect Technology Conference, IEEE, Piscataway, NY, 1008, pp. 233.

4. J.M. Paik, K.C. Park and Y.C. Joo, J. Elec Mails. 33, 1, 48 (2004).

5. L. L. Vanasupa, Y. C. Joo, P. R. Besser, and S. Pramanick, J. Appl Phys 85, 5, 2583 (1999).

6. B. C. Valek, J. C. Bravman, N. Tamura, A. A. MacDowell, R. Celestre, H. Padmore, R. Spolenak, W. L. Brown, B. W. Batterman and J. R. Patel, Appl Phys Lett. 81, 4168 (2002).

7. B. C. Valek, N. Tamura, R. Spolenak, W. A. Caldwell, A. MacDowell, R. S. Celestre, H. A. Padmore, J. C. Bravman, B. W. Batterman, W. D. Nix, and J. R. Patel, J. Appl Phys 94, 3757 (2003).

8. Arief S. Budiman, N. Tamura, B. C. Valek, K. Gadre, J. Maiz, R. Spolenak, W. A. Caldwell, W. D. Nix and J. R. Patel. Unexpected Mode of Plastic Deformation in Cu Damascene Lines Undergoing Electromigration, Mater. Res. Soc. Symp. Proc, 2004, pp. 812.

9. Arief S. Budiman, N. Tamura, B. C. Valek, K. Gadre, J. Maiz, R. Spolenak, J. R. Patel, W. D. Nix. Electromigration-Induced Plastic Deformation in Cu Damascene Interconnect Lines as Revealed by Synchrotron X-Ray Microdiffraction, Mater. Res. Soc. Symp. Proc, 2006, pp. 914.

1O.N. Tamura, A. A. MacDowell, R. Spolenak, B. C. Valek, J. C. Bravman, W. L. Brown, R. S. Celestre, H. A. Padmore, B. W. Batterman and J. R. Patel, J. SynchrotronRad. 10, 137-143 (2003).

1 l.R. W. Cahn, J. Inst Met, 86, 121 (1949).

12.J. F.Nye,^ctaMeta//., 1, 153 (1953). 